\title{
Effect of sample volume on yield of positive blood cultures from adult patients with haematological malignancy
}

\author{
D F J Brown, R E Warren
}

\begin{abstract}
Six hundred and sixteen blood samples from patients with haematological malignancy were each distributed equally among three identical cells in a Malthus Microbiological Growth Analyser. The mean (SD) volumes inoculated into sets in which one, two, or three of the three bottles were positive were $37 \cdot 7(10 \cdot 1) \mathrm{ml}$, $37.4(12.9) \mathrm{ml}$, and $37.7(10.5) \mathrm{ml}$, respectively. Overall, clinically important organisms were isolated from one bottle only with 18 cultures, from two bottles only with 19 cultures, and from all three bottles in a set with 104 cultures. If the yield from a single bottle inoculated with a mean volume of $12.6 \mathrm{ml}$ blood is taken as $100 \%$, the yield from $25.2 \mathrm{ml}$ in two bottles was $110.7 \%$ and the yield from $37.7 \mathrm{ml}$ in three bottles was $115.6 \%$. The increased yield from increased volume was considerably lower than that reported from unselected groups of patients, which suggests that the magnitude of bacteriaemia is greater in patients with neutropenia. The isolation of infecting organisms from the blood of patients with neutropenia is, however, particularly important in directing chemotherapy and consequently $45 \mathrm{ml}$ blood samples from these patients continue to be requested.
\end{abstract}

In adults around $40 \%$ of blood samples taken from peripheral veins for culture and assessed by pour plate or lysis methods contain less than one colony forming unit (cfu) per $\mathrm{ml}^{1-7}$ It might therefore be expected that the isolation rate from blood cultures would increase significantly with the volume of the blood sample, and work with a variety of blood culture systems with various media has shown this to be the case. ${ }^{8-16}$ These studies have shown an increase in the isolation rate with increasing volume of blood sample up to 30 $\mathrm{ml}$. With samples greater than $30 \mathrm{ml}$ there is little information available.

Patients with haematological malignancy have a high risk of bacteraemia when neutropenic. Blood cultures are usually taken when the patient has been feverish for two hours or less and immediately before chemotherapy is given. Consequently clinical illness is at a defined early stage. The reduced number of phagocytic cells in neutropenia might affect the numbers of organisms present in blood samples taken for culture when compared with other groups of patients. Larger numbers of organisms are present in the blood of patients with bacteraemia whose spleens have been removed, ${ }^{17}$ and the same might be expected with neutropenic patients. Alternatively, the higher risk of bacteraemia in neutropenic patients, and earlier treatment, may result in low level bacteraemia occurring more often. In one study of bone marrow transplant recipients $62 \%$ of blood cultures had organisms present at less than $1 \mathrm{cfu} / \mathrm{ml}^{18}$ We investigated the effect of blood sample volume on the isolation rate of clinically important organisms from patients with haematological malignancy and neutropenia.

\section{Methods}

BLOOD CULTURE PROCEDURE

Between October 1986 and December 1987 clinicians were requested to take $45 \mathrm{ml}$ of blood at a single venepuncture from adult patients with haematological malignancy. Patients with clinically important isolates were included in the analysis if their neutrophil counts were $<1.0 \times 10^{9} / 1$ when blood cultures were taken. Blood cultures were taken when the patient was feverish (temperature higher than $38^{\circ} \mathrm{C}$ on two occasions or greater than $38.5^{\circ} \mathrm{C}$ on one occasion), hypotensive, or failing to respond to antimicrobial agents already administered.

Cultures from central lines and peripheral veins were not distinguished by clinicians. Blood samples were divided equally among three identical Malthus conductivity cells, ${ }^{19}$ each containing $85 \mathrm{ml}$ of medium. The medium was supplemented brain heart infusion broth as described previously, ${ }^{19}$ except that the saponin was replaced by $0.5 \%$ weight/volume dextran (Sigma D5251). The dextran eliminates the early signal from blood which may prevent detection of early signals from bacteria. ${ }^{20}$ All bottles were weighed before distribution from the laboratory and, after inoculation with a blood sample, on receipt back in the laboratory. As blood has a density of about 1.055 , blood volumes could be estimated from the weight of the samples. The blood cultures were monitored continuously on the Malthus apparatus and processed as described previously. ${ }^{1921}$ The medium, atmosphere of incubation, and screening procedure was identical for all three bottles in each set. 
CRITERIA FOR DETERMINING CLINICAI IMPORTANCE OF ISOLATES

All patients had clinical features of systemic infection and were neutropenic. None was judged to be clinically important on bacteriological criteria alone. Isolates commonly found as contaminants in blood cultures-that is, coagulase negative staphylococci, Bacillus spp and diphtheroid organisms-were considered to be clinically important if there was evidence of line or tunnel infection with the same organism, if a cannula tip on removal yielded more than 15 colony forming units, ${ }^{22}$ or if the same organism was isolated from more than one blood culture bottle in a set or from more than one set over 24 hours.

\section{Results}

COMPLIANCE WITH REQUEST FOR 45 ML BLOOD SAMPLES

Six hundred and sixteen blood cultures were examined. Clinically important organisms were isolated from 141 blood cultures and data on volumes of blood inoculated into bottles were available for 108 of these. The mean (SD) total volumes of blood inoculated into sets in which one, two, or three bottles were positive were $37 \cdot 7(10 \cdot 1) \mathrm{ml}, 37 \cdot 4(12.9) \mathrm{ml}$, and 37.7 $(10.5) \mathrm{ml}$, respectively. There was reasonable compliance with the request for $45 \mathrm{ml}$ samples and there was no evidence of bias in volumes of blood inoculated into sets in which different numbers of bottles were positive. In sets where only one or two of the three bottles were positive the mean (SD) volumes inoculated into positive and negative bottles were 12.6 (4.6) $\mathrm{ml}$ and $12.5(3.9) \mathrm{ml}$, respectively, indicat- ing equal distribution of blood among bottles in a set.

ISOLATES FROM BLOOD CULTURES

The isolates from only one out of three bottles, from two out of three bottles, and from all three bottles in a set are shown in the table. In total, clinically important organisms were isolated from one bottle only in 18 cultures ( 20 isolates), from two bottles only in 19 cultures (21 isolates), and from all three bottles in 104 cultures (114 isolates). Ten of the 141 positive cultures were polymicrobial and from these 10 cultures two isolates were from one bottle only, two were from two bottles only, and 20 were from all three bottles.

\section{Discussion}

In our study the effect of blood sample volume on the yield of positive cultures may be estimated by comparing the yield of positive cultures from one, two, or three identical bottles in a set in which each of the bottles was inoculated with a similar volume of blood.

The yield from one bottle may be calculated as one third of the cultures positive in a single bottle, plus two thirds of the cultures positive in two bottles, plus all cultures positive in three bottles-that is, $6+12+104=122$. Similarly, the yield from two bottles is two thirds of the cultures positive in a single bottle plus all cultures positive in two and three bottles-that is, $12+19+104=135$; and the yield from all three bottles is the number of cultures positive in any bottle-that is, $18+19$ $+104=141$. A mean volume of $12.6 \mathrm{ml}$ blood was added to each bottle in a set. Hence if the yield from culture of $12.6 \mathrm{ml}$ in one bottle was

Isolates from blood cultures

\begin{tabular}{|c|c|c|c|}
\hline \multirow[b]{2}{*}{ Organisms isolated } & \multicolumn{3}{|c|}{ Number with isolates from: } \\
\hline & One bottle & Two bottles & Three bottles \\
\hline $\begin{array}{l}\text { Escherichia coli } \\
\text { Klebsiella sp } \\
\text { Enterobacter sp } \\
\text { Citrobacter sp } \\
\text { Salmonella sp } \\
\text { Acinetobacter sp } \\
\text { Pseudomonas sp } \\
\text { Campylobacter sp } \\
\text { Capnocytophaga ochracea } \\
\text { Staphylococcus aureus } \\
\text { Coagulase negative staphylococci } \\
\text { Streptococcus pneumoniae } \\
\text { Streptococcus mitis, sanguis, salivarius } \\
\text { Streptococcus faecalis } \\
\text { Streptococcus faecium } \\
\text { Group A or C streptococci } \\
\text { Listeria monocytogenes } \\
\text { Unidentified diphtheroid organism } \\
\text { Bacillus cereus } \\
\text { Clostridium sp } \\
\text { Fusobacterium necrophorum } \\
\text { Escherichia coli and Klebsiella sp (and Clostridium tertium in two bottles) } \\
\text { Escherichia coli and Klebsiella sp (and Streptococcus sanguis in one bottle) } \\
\text { Escherichia coli and Streptococcus faecium (and Clostridium tertium in two bottles) } \\
\text { Escherichia coli and Streptococcus sanguis } \\
\text { Klebsiella sp and Streptococcus sanguis } \\
\text { Enterobacter sp Streptococcus sanguis } \\
\text { Pseudomonas sp and Staphylococcus epidermidis } \\
\text { Staphylococcus aureus (and Escherichia coli in one bottle) } \\
\text { Streptococcus faecium and Staphylococcus epidermidis } \\
\text { Clostridium sp and Streptococcus mitis and Bacillus sp } \\
\text { Total Gram negative monomicrobials } \\
\text { Total Gram positive monomicrobials } \\
\text { Total anaerobic monomicrobials } \\
\text { Total polymicrobials } \\
\text { Overall totals }\end{array}$ & $\begin{array}{r}6 \\
1 \\
1 \\
0 \\
0 \\
0 \\
1 \\
0 \\
0 \\
3 \\
2 \\
0 \\
0 \\
1 \\
0 \\
0 \\
0 \\
0 \\
0 \\
2 \\
1 \\
0 \\
0 \\
0 \\
0 \\
0 \\
0 \\
0 \\
0 \\
0 \\
0 \\
0 \\
0 \\
9\end{array}$ & $\begin{array}{r}3 \\
1 \\
0 \\
0 \\
1 \\
0 \\
1 \\
0 \\
1 \\
0 \\
6 \\
0 \\
3 \\
2 \\
0 \\
0 \\
1 \\
0 \\
0 \\
0 \\
0 \\
0 \\
0 \\
0 \\
0 \\
0 \\
0 \\
0 \\
0 \\
0\end{array}$ & $\begin{array}{r}21 \\
4 \\
4 \\
2 \\
0 \\
1 \\
8 \\
2 \\
0 \\
9 \\
22 \\
1 \\
6 \\
3 \\
2 \\
2 \\
0 \\
1 \\
1 \\
5 \\
0 \\
1 \\
1 \\
1 \\
1 \\
1 \\
1 \\
1 \\
1 \\
1 \\
1 \\
42 \\
47 \\
5 \\
10 \\
104\end{array}$ \\
\hline
\end{tabular}


taken as $100^{\circ}{ }_{0}$ then the yield from $25.2 \mathrm{ml}$ in two bottles was $110 \cdot 7 \%$ and the yield from $37 \cdot 7$ $\mathrm{ml}$ in three bottles was $115.6 \%$. Ilstrup and Washington found that the yields from culture of $20 \mathrm{ml}$ in two bottles and $30 \mathrm{ml}$ in three bottles compared with $10 \mathrm{ml}$ in one bottle were $138 \%$ and $162 \%$, respectively. ${ }^{10}$ In our study the increased yield with increased volume was substantially lower. The inference is that the magnitude of bacteraemia in patients with neutropenia, managed with indwelling catheters, is greater than in other groups of patients. This could be directly studied with a quantitative blood culture system.

Assessment of the clinical importance of seven isolates of coagulase negative staphylococci and streptococci was based on their isolation from more than one of three bottles in a set. This is a different criterion than would be applied to isolates of such organisms from most groups of patients, but necessary with these patients as early treatment before repeat culture is essential. The criterion of isolation from more than one bottle in a set is affected by the number of bottles in the set and some of the seven isolates might be judged not to be clinically important if a two bottle set had been used. Even if these isolates were eliminated from our assessment, however, the increased isolation rate with increased volume would change only marginally and our conclusions would not be affected.

In unselected patients low numbers of organisms in blood are more common with Gram negative bacteraemias. ${ }^{1-7}$ There is also evidence that the number of bacteria present in samples drawn from intravascular cannulae is greater than that from peripheral vein samples in patients with lines infected with Gram positive organisms. ${ }^{23-26}$ Sepsis associated with cannulae in our unit is common and is also usually caused by Gram positive organisms. Isolation of $\mathrm{Gram}$ negative bacilli from a single bottle would therefore be expected to be much more common than isolation of Gram positive organisms from a single bottle. Our overall results with neutropenic patients do not show this difference, despite the probable inclusion of some cultures taken using intravascular devices. It may be that higher counts of Gram negative bacteria in the blood are more common in patients with haematological malignancy and neutropenia than in unselected patients. The benefit to be gained from increasing the volume of blood cultured is consequently reduced.

In polymicrobial bacteraemia in our study the increase in isolation rate with increasing volume was less evident than with monomicrobial bacteraemia, suggesting that the numbers of organisms in the blood are greater in polymicrobial bacteraemia. The higher mortality in neutropenic patients with polymicrobial bacteraemia is well recognised. ${ }^{27}$

In this study the rate of isolation of clinically important organisms increased with increased volume of blood sample up to $37.7 \mathrm{ml}$, but the increase seemed to be less than that reported for unselected groups of patients. The isolation of infecting organisms from the blood of patients with neutropenia is, however, particularly important in directing chemotherapy and consequently we continue to request $45 \mathrm{ml}$ blood samples from these patients.

We are grateful for the assistance of the staff on the Haematology ward at Addenbrooke's Hospital, Cambridge.

1 Brannon P, Kiehn TE. Large-scale clinical comparison of the lysis-centrifugation and radiometric systems for blood culture. J Clin Microbiol 1985;22:951-4.

2 Dorn GL, Burson GG, Haynes JR. Blood culture technique based on centrifugation: Clinical evaluation. J Clin Microbiol 1976;3:258-63.

3 Dorn GL, Land GA, Wilson GE. Improved blood culture technique based on centrifugation: Clinical evaluation. J Clin Microbiol 1979;9:391-6.

4 Henry NK, McLimans CA, Wright AJ, Thompson RL, Wilson WR, Washington JA. Microbiological and clinical evaluation of the isolator lysis-centrifugation blood culture tube. J Clin Microbiol 1983;17:864-9.

5 Hillberry SM. Use of a centrifugation method of blood culturing in a community acute-care hospital. Lab Med culturing in a

6 Sullivan NM, Sutter VL, Finegold SM. Practical aerobic membrane filtration blood culture technique: Clinical blood culture trial. J Clin Pathol 1975;1:37-43.

7 Washington JA. Characteristics of bacteremia relevant to its laboratory diagnosis. In Washington JA, ed. The detection of septicemia. West Palm Beach: CRC Press, 1978:27-30.

8 Fox, H, Leaman WG. Observation on blood cultures with special reference to the quantity of the blood used. $J \mathrm{Lab}$ Clin Med 1926;11:145-50.

9 Hall MM, Ilstrup DM, Washington JA. Effect of volume of blood cultured on detection of bacteremia. J Clin Microbiol 1976;3:643-5.

10 Ilstrup DM, Washington JA. The importance of volume of blood cultured in the detection of bacteremia and funblood cultured in the detection of bacteremia a
gemia. Diag Microbiol Infect Dis 1983;1:107-10.

11 Plorde JJ, Tenover FC, Carlson LG. Specimen volume versus yield in the BACTEC blood culture system. J Clin Microbiol 1985;22:292-5.

12 Sandven P, Hoiby A. The importance of blood volume cultured on detection of bacteraemia. Acta Pathol Microbiol Scand (Sect B) 1981;89:149-52.

13 Shanson DC, Thomas F, Wilson D. Effect of volume of blood cultured on detection of streptococcus viridans bacteraemia. J Clin Pathol 1984;37:568-70.

14 Tenney JH, Reller B, Mirrett S, Wang WL, Weinstein MP. Controlled evaluation of the volume of blood cultured in detection of bacteremia and fungemia.J Clin Microbiol detection of bacterc

15 Washington JA. Conventional approaches to blood culture. In: Washington JA, ed. The detection of septicemia. West Palm Beach: CRC Press, 1978:41-87.

16 Washington JA, Ilstrup DM. Blood cultures: Issues and controversies. Rev Infect Dis 1986;8:792-802.

17 Torres J, Bisno AL. Hyposplenism and pneumococcemia: Visualisation of Diplococcus pneumoniae in the peripheral blood smear. Am J Med 1973;55:851-5.

18 Hamilton DJ, Amos D, Schwartz RW, Dent CM, Counts $\mathrm{GW}$. Effect of delay in processing on lysis centrifugation blood culture results from marrow transplant patients. J Clin Microbiol 1989;27:1588-93.

19 Brown DFJ, Warner M, Taylor CED, Warren RE. Automated detection of micro-organisms in blood cultures by means of the Malthus Microbiological Growth tures by means of the Malthus Micro
Analyser. J Clin Pathol 1984;37:65-9.

20 Curtis GDW, Thomas CD, Johnston HH A note on the use of dextran in blood cultures monitored by conductance methods. J Appl Bacteriol 1985;58:571-5.

21 Brown DFJ, Warner M, Taylor CED, Warren RE. Automated screening of blood cultures with the Malthus microbiological growth analyser. J Clin Pathol 1988;41: 671-5.

22 Maki DG, Weise CE, Sarafin HW. Semiquantitative culture method for identifying intravenous-catheter related infection. N Engl J Med 1977;296:1305-9.

23 Andremont A, Paulet R, Nitenberg G, Hill C. Value of semiquantitative cultures of blood drawn through catheter hubs for estimating the risk of catheter tip colonization in hubs for estimating the risk of catheter tip colonizati

24 Paya CV, Guerra L, Marsh HM, Farnell MB, Washington JA, Thompson RL. Limited usefulness of quantitative culture of blood drawn through the device for diagnosis of intravenous-device-related bacteremia. J Clin Microbiol 1989;27:1431-3.

25 Raucher HS, Hyatt AC, Barzilai A, et al. Quantitative blood cultures in the evaluation of septicaemia in children with Broviac catheters. J Pediatr 1984;104:29-33.

26 Wing EJ, Norden CW, Shadduck RK, Winkelstein A. Use of quantitative bacteriologic techniques to diagnose catheter-related sepsis. Arch Intern Med 1979;139:482-3.

27 Whimbey E, Kiehen TE, Brannon P, Blevins A, Armstrong $D$. Bacteremia and fungemia in patients with neoplastic disease. $A m J$ Med 1987;82:223-9. 